\title{
Pitfalls, prevention, and treatment of hyperuricemia during tumor lysis syndrome in the era of rasburicase (recombinant urate oxidase)
}

\author{
Andrea Pession \\ Fraia Melchionda \\ Claudia Castellini \\ Oncologia Ematologia Pediatrica \\ "Lalla Seràgnoli", Clinica Pediatrica, \\ Università degli Studi di Bologna, \\ Bologna, Italy
}

\begin{abstract}
Along with hydration and urinary alkalinization, allopurinol has been the standard agent for the management of hyperuricemia in patients with a high tumor burden at risk of tumor lysis syndrome; however, this agent often fails to prevent and treat this complication effectively. Rasburicase (recombinant urate oxidase) has been shown to be effective in reducing uric acid and preventing uric acid accumulation in patients with hematologic malignancies with hyperuricemia or at high risk of developing it. Rasburicase acts at the end of the purine catabolic pathway and, unlike allopurinol, does not induce accumulation of xanthine or hypoxanthine. Its rapid onset of action and the ability to lower pre-existing elevated uric acid levels are the advantages of rasburicase over allopurinol. Rasburicase represents an effective alternative to allopurinol to promptly reduce uric acid levels, improve patient's electrolyte status, and reverse renal insufficiency. The drug, initially studied in pediatric patients with acute lymphoblastic leukemia and aggressive non-Hodgkin lymphoma, seems to show comparable benefit in adults with similar lymphoid malignancies or at high risk of tumor lysis syndrome. Current and future trials will evaluate alternative doses and different schedules of rasburicase to maintain its efficacy while reducing its cost. The review provides a comprehensive and detailed review of pathogenesis, laboratory, and clinical presentation of TLS together with clinical studies already performed both in pediatric and adult patients.
\end{abstract}

Keywords: tumor lysis syndrome, urate oxidase, rasburicase, allopurinol, uric acid

\section{Tumor lysis syndrome}

Tumor lysis syndrome (TLS) is a severe and potentially life-threatening complication in patients with cancer; it may occur spontaneously (sTLS) or, more frequently, as a consequence of chemotherapy, radiotherapy, or immunotherapy (Flombaum 2000; Locatelli and Rossi 2005; Pession and Barbieri 2005; Riccio et al 2006). It was first reported in 1929 in a group of adults with chronic leukemia (Bedrna 1929). Since then, several studies on TLS have been published, but its incidence is still not precisely defined (Flombaum 2000; Turtureanu-Hanganu 2002).

Recently Cairo and Bishop proposed a uniform definition of TLS, identifying a laboratory TLS (LTLS) and clinical TLS (CTLS). This stratification of TLS may provide a useful tool to better identify appropriate therapy. LTLS is defined by the presence of at least 2 of the following laboratory data: hyperuricemia, hyperkalemia, hyperphosphatemia, and secondary hypocalcemia, defined as $25 \%$ change from baseline or threshold value of serum level (Table 1). CTLS is defined by the presence of LTLS and one or more of the most significant clinical complications related to TLS: renal insufficiency, cardiac arrhythmias/sudden death, and seizure (Cairo and Bishop 2004). Not surprisingly, laboratory evidence of TLS has been reported much more frequently than the symptomatic clinical syndrome (Jasek and Day 1994). 
Table I Laboratory criteria of tumor lysis syndrome

\begin{tabular}{|c|c|}
\hline Uric acid & $\begin{array}{l}\text { Increase of more than } 25 \% \text { from baseline if available or } \\
\text { Values } \geq 476 \mu \mathrm{mol} / \mathrm{L} 8 \mathrm{mg} / \mathrm{dL}\end{array}$ \\
\hline Potassium & $\begin{array}{l}\text { Increase of more than } 25 \% \text { from baseline if available or } \\
\text { Values } \geq 6.0 \mathrm{mmol} / \mathrm{L} 6 \mathrm{mEq} / \mathrm{dL}\end{array}$ \\
\hline Phosphorus & $\begin{array}{l}\text { Increase of more than } 25 \% \text { from baseline if available or } \\
\text { Values } \geq 1.45 \mathrm{mmol} / \mathrm{L} 4.5 \mathrm{mg} / \mathrm{dL} \text { for adults patients } \\
\text { Values } \geq 2.1 \mathrm{mmol} / \mathrm{L} 6.5 \mathrm{mg} / \mathrm{dL} \text { for pediatric patients }\end{array}$ \\
\hline Calcium & $\begin{array}{l}\text { Decrease of more than } 25 \% \text { from baseline if available } \\
\text { or Values } \geq 1.75 \mathrm{mmol} / \mathrm{L} 7 \mathrm{mg} / \mathrm{dL}\end{array}$ \\
\hline
\end{tabular}

Modified from Cairo and Bishop (2004).

TLS may also be a spontaneous event (sTLS) occurring prior to chemotherapy initiation in a variety of malignancies, which has been reported mostly in patients with Burkitt's lymphoma or mature B-cell leukemia, both characterized by a very rapid cell turnover rates (Alkhuja and Ulrich 2002). Identifying patients at risk of sTLS may have a significant impact on outcome. In a recent study, sTLS in patients with malignancies is defined by presence of pretreatment hyperuricemic acute renal failure (ARF), ratio of urinary uric acid to creatinine $(\mathrm{Cr})>1.0$, and significantly elevated lactate dehydrogenase ( $>500$ units/L); TLS was demonstrated to occur in $1.08 \%$ of 926 patients with ARF (Hsu et al 2004). A possible distinction between spontaneous tumor lysis and TLS occurring after therapy is the lack of hyperphosphatemia in the spontaneous form.

Factors predisposing the development of TLS include a high tumor growth fraction, as in Burkitt's lymphoma, lymphoblastic lymphoma, B-cell acute lymphoblastic leukemia (B-ALL), and T-cell ALL with hyperleukocytosis and/or extensive extramedullary disease; cases of TLS in patients with solid tumor have also been extensively reported in tumors with high sensitivity to chemotherapy and large tumor burden (Feld et al 2000; Habib and Saliba 2002; Stark et al 1987; Tomlinson and Solberg 1984). In addition elevated lactate dehydrogenase (LDH) serum levels, and decreased urinary flow confer higher risk of TLS onset (Cairo and Bishop 2004). Although TLS occurs at all ages, advanced age leading to impaired renal function may predispose patients to clinically significant TLS. Neither racial nor sex predilection has been reported.

Cytotoxic therapy or highly proliferative tumor is the primary origin of TLS. Some chemotherapeutic agents such as cytosine arabinoside, etoposide, cisplatin, and intrathecal methotrexate have been more frequently implicated in causing TLS (Locatelli and Rossi 2005), although no one regimen or drug may be specifically indicated as carrying a higher likelihood of causing TLS. Thus host characteristics remain key factors in TLS development. TLS has been also observed after infusion of monoclonal antibodies (rituximab, gemtuzumabozogamicin), high dose-corticosteroids, radiotherapy (total body irradiation), interferon, thalidomide, hydroxyurea, imatinib, and fludarabine. Anecdotal cases of TLS after hormonal therapy for breast carcinoma and prostate cancer have also been reported. There is no consensus to date for stratification of patients at high risk of TLS, but data can be drawn from most of the reports available to suggest some useful criteria (for adults and children), as indicated in Table 2. TLS onset occurs early in patients with high risk hematological neoplasm (within 1-5 days of therapy initiation), due to peculiar biological characteristics of malignant lymphoid cells (high proliferative rate, synchronous kinetics); thus careful monitoring of laboratory parameters and supportive therapies are the most important measures to prevent or successfully manage TLS. In contrast, TLS occurring in solid tumor patients usually shows a later onset than in hematological malignancies. This difference may be related to more synchronous kinetics of lymphomyeloproliferative disorders cells than solid tumor cell. TLS is a rare but extremely severe complication of treatment of adult solid tumors. TLS toxicity-related death rate is surprisingly higher in this subset of patients, in which nearly $35 \%$ of all cases developing clinical TLS die. The reasons might lie in the delayed initiation of prophylactic measures to prevent or treat TLS in patients with solid tumor, and generally less close monitoring during and after therapy initiation. Virtually all tumor types have been reported including some diseases relatively or poorly responsive to chemotherapy (melanoma, colorectal, gastric, vulvar, hepatocellular carcinoma), but the majority of reported cases of TLS were in patients with cancer known to be highly or very highly sensitive to chemotherapy such as small cell lung cancer and germ cell carcinoma (Sparano et al 1990; Coutinho et al 1997; Habib and Saliba 2002; Annemans et al 2003).

In a recent paper Wossmann et al analyzed the incidence and complications of TLS in 1791 children with non-Hodgkin lymphoma (NHL) enrolled in the two subsequent multicenter studies NHL-BFM 90 and 95. Out of this group, 78 (4.4\%) developed a TLS and 42 (2.3\%) oligoanuria. Patients with Burkitt's lymphoma or B-ALL had a higher incidence of TLS (8.4\%) and anuria (4.4\%); in particular, patients with B-ALL had the highest risk to develop a TLS (26.4\%) and anuria (14.1\%). Of the 790 patients with Burkitt's lymphoma or B-ALL, tumor burden, as indicated by the plasma level of $\mathrm{LDH}$, was the main predictor for the development of both TLS and anuria. The TLS incidence was 1.2\%, 12.7\%, and 19.1\% 
Table 2 Risk stratification definition and criteria

At high risk of developing tumor lysis syndrome are those patients who carry at least one of the following co-morbidities, disease-related or therapy-related factors

\begin{tabular}{|c|c|}
\hline & Pediatric patients \\
\hline Co-morbidities & $\begin{array}{l}\text { - Renal impairment at diagnosis appear to be } \\
\text { the only relevant co-morbidity in children } \\
\text { - Kidney tumor involvement at onset } \\
\text { - Hyperuricemia UA }>8 \mathrm{mg} / \mathrm{dL}\end{array}$ \\
\hline $\begin{array}{l}\text { Disease-related } \\
\text { factors }\end{array}$ & $\begin{array}{l}\text { - Tumors with high proliferative rate } \\
\text { - Bulky solid tumor especially if massive liver } \\
\text { metastasis } \\
\text { - Lympho-myeloproliferative disorders with } \\
\text { large tumor burden as WBC }>50,000 / \mathrm{mm}^{3} \text { or } \\
\text { LDH }>2 \text { times normal level } \\
\text { - High grade lymphomas } \\
\text { particularly Burkitt's lymphoma and T-cell NHL } \\
\text { - B-cell and T-cell acute lymphoblastic leukemias }\end{array}$ \\
\hline $\begin{array}{l}\text { Treatment-related } \\
\text { factors }\end{array}$ & $\begin{array}{l}\text { - Intensive cytoreductive chemotherapy in } \\
\text { hematologic malignancies } \\
\text { Adult patients }\end{array}$ \\
\hline Co-morbidities & $\begin{array}{l}\text { - Dehydratation } \\
\text { - Hyponatriemia } \\
\text { - Pre-existing renal impairment } \\
\text { including tumor infiltration that reduce renal } \\
\text { function } \\
\text { - Obstructive uropathy } \\
\text { - Hyperuricemia UA }>10 \mathrm{mg} / \mathrm{dL}\end{array}$ \\
\hline $\begin{array}{l}\text { Disease-related } \\
\text { factors }\end{array}$ & $\begin{array}{l}\text { - Tumors with high proliferative rate } \\
\text { - Bulky solid tumor especially if massive } \\
\text { liver metastasis } \\
\text { - Lympho-myeloproliferative disorders with } \\
\text { large tumor burden such as }>50,000 / \mathrm{mm}^{3} \text { or } \\
\text { LDH }>2 \text { times normal level } \\
\text { - Advanced germ cell lung cancer } \\
\text { - High grade lymphomas particularly Burkitt's } \\
\text { lymphoma, a T-cell NHL } \\
\text { - B-cell acute lymphoblastic leukemias }\end{array}$ \\
\hline $\begin{array}{l}\text { Treatment-related } \\
\text { factors }\end{array}$ & $\begin{array}{l}\text { - Intensive cytoreductive chemotherapy in } \\
\text { hematologic malignancies }\end{array}$ \\
\hline
\end{tabular}

Abbreviations: $\mathrm{LDH}$, lactate dehydrogenase; $\mathrm{NHL}$, non-Hodgkin lymphoma; UA, uric acid; WBC, white blood cell.

for patients with a LDH level of $<500 \mathrm{U} / \mathrm{L}, 500-1000 \mathrm{U} / \mathrm{L}$, and $>1000 \mathrm{U} / \mathrm{L}$ respectively. The incidence of TLS in patients with T-lymphoblastic lymphoma and other B-cell NHL was below $2 \%$ and anuria occurred in less than $1 \%$ (Wossmann et al 2003). TLS is rare in acute myeloid leukemia (AML) despite the high blood cell count. TLS has also been documented in childhood solid tumors such as neuroblastoma, medulloblastoma, and germ cell tumors. TLS has also been observed, although rarely, in children treated with agents without a potent cytotoxic action such as interferon alfa, IL-2, STI-571.

\section{Pathogenesis of TLS}

Pathogenesis of TLS is complex and derives from rapid turnover and/or large destruction of tumor cells, resulting in a synchronous release of intracellular ions and metabolic byproducts into the circulation. When this massive discharge cannot be compensated for by cellular buffering and renal elimination mechanisms, numerous life-threatening metabolic derangements occur such as hyperuricemia, hyperkalemia, hypocalcemia hyperphosphatemia, metabolic acidosis, and acute renal insufficiency (Figure 1).

\section{Hyperuricemia}

The increase in uric acid concentration in plasma is constantly present in patients with TLS; hyperuricemia may be already present at time of diagnosis or develop 2-3 days after initiation of anti-cancer treatment. A high nucleic acid content and a very active purine metabolism are characteristic of tumor cells. The metabolism of nucleic acid purines (adenosine and guanine) occurs in the liver, where purines are reduced to xanthine, and then degraded to uric acid by the enzyme xanthine oxidase. Thus the destruction of tumor cells yields a large amount of uric acid. Some animal species are known to have the enzyme urate oxidase which catalyzes degradation of uric acid to allantoin, a much more soluble compound. Unfortunately during evolution humans and non-human primates have lost this catabolic pathway due to a nonsense mutation of this enzyme (Yeldandi et al 1991). The presence of hyperuricemia may induce the crystallization of uric acid

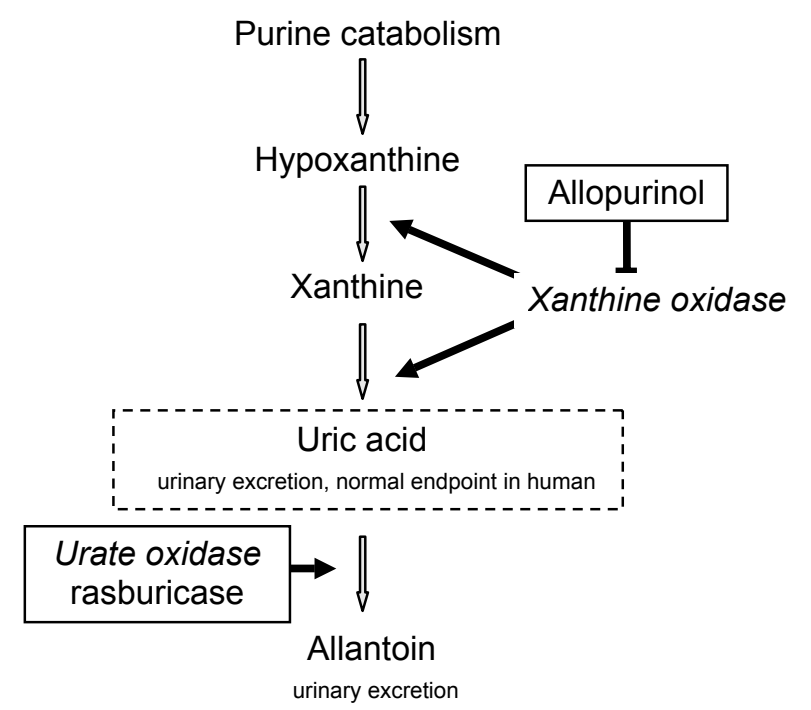

$\overline{\text { Barred lines=inhibition. Arrows=activation or consequences }}$

Figure I Purine catabolic pathway and site of action of allopurinol and rasburicase. 
in the renal collecting ducts and in the deep cortical and medullar vessels, leading to the development of acute renal failure. The crystallization of uric acid is also enhanced by hemoconcentration and decreased tubular flow rate; moreover, a reduced solubility of uric acid is enhanced by decreased $\mathrm{pH}$ of renal tubule.

\section{Hyperkalemia}

Hyperkalemia results from massive cellular degradation and efflux of potassium, and may represent a life-threatening complication in patients with TLS. The presence of renal insufficiency and metabolic acidosis may worsen hyperkalemia, overwhelming the potassium excretory capacity of the kidney. Clinical manifestations of hyperkalemia are nausea, vomiting, and diarrhea. More specifically hyperkalemia induces cardiac and neuromuscular abnormalities such as electrocardiographic (ECG) changes and life-threatening cardiac arrhythmia, including asystole. When hyperkalemia is severe it is always associated with ECG alterations such as peaked $\mathrm{T}$ waves, flattened $\mathrm{P}$ waves, prolonged $\mathrm{PR}$ interval, widened QRS complexes, deep S waves and sine waves, ventricular tachycardia or fibrillation, syncope, and possible sudden death. These ECG abnormalities must be promptly diagnosed and treated through stabilization of the myocardial cell membrane, intracellular shift of potassium, and reduction of total ion load, in order to reduce the risk of fatal ventricular arrhythmia. Neuromuscular signs of hyperkalemia includes muscle weakness, paresthesias, and cramps (Parham et al 2006).

\section{Hyperphosphatemia and hypocalcemia}

As with the other laboratory abnormalities, hyperphosphatemia is the consequence of rapid cell lysis; it develops quickly, 24-48 hours after anti-cancer treatment. In this regard, it has been estimated that the phosphorus content of lymphoblasts is 3-4 times that of normal lymphocytes (Barton 1989; Locatelli and Rossi 2005). In order to reduce phosphorus concentration the kidney decreases tubular re-adsorption and increases excretion. Hyperphosphatemia may be also induced by acute renal insufficiency. Severe hyperphosphatemia may be associated with nausea and vomiting, lethargy, and seizure.

Hypocalcemia usually occurs together with hyperphosphatemia, due to precipitation of calcium phosphate in soft tissues including kidney, where it produces nephrocalcinosis, which may occur when in vivo concentration of calcium phosphate salts exceeds the solubility product of $4.6 \mathrm{mmol} / \mathrm{L}$. Moreover, deposits of calcium in the renal tubules elicits an inflammatory response exacerbating the risk of ARF (Ribeiro and Pui 2003; Locatelli and Rossi 2005). Calcium phosphate is less soluble at an alkaline $\mathrm{pH}$, which can exacerbate the risk of its deposit. Iatrogenic urine alkalinization in patients presenting TLS with hyperphosphatemia and/or hypocalcemia may be associated with an increased risk of nephrocalcinosis, and thus it must be avoided especially in patients whose calcium $\times$ phosphate product exceeds the solubility limit. When hypocalcemia is severe it may be associated with critical clinical manifestation such as muscle cramps, paresthesias, tetany and seizures, cardiac ECG abnormalities such as lengthening of QT interval, ventricular arrythmias, and hypotension. Treatment of asymptomatic hypocalcemia is not generally recommended in patients with TLS due to the higher risk calcium phosphate precipitation and consequently nephrocalcinosis.

\section{Metabolic acidosis}

Metabolic acidosis may develop concomitant with ARF and because of liberation of a large amount of endogenous intracellular metabolites from cellular catabolism. Acidosis induces a decrease in bicarbonate concentration and an high anion gap which may worsen the imbalance of electrolytes in patients with TLS, such as uric acid solubility and impaired intracellular uptake of potassium (Filler 2001).

\section{Acute renal failure}

As mentioned previously the kidney is the primary organ involved in clearance of uric acid, potassium, and phosphate; thus, it is expected that abnormalities of renal function, characteristic of TLS, predispose patients to worsening of metabolic derangements typical of this syndrome. Renal dysfunction can be severe in patients with TLS, but in the majority of cases, if diagnosed in a timely manner and promptly treated with supportive measures, it is reversible. The major cause of oliguric acute renal failure is the nephropathy mediated by mechanical obstruction by uric acid crystals (Hsu and Huang 2004). Intra-tubular obstruction causes azotemia/oliguria in acute uric acid nephropathy, and vascular obstruction can contribute to filtration failure. Uric acid crystallization in the distal tubules/cortical collecting ducts is not the only factor operating in the mechanical obstruction of kidney. In fact, as mentioned above, nephrocalcinosis significantly contributes to renal injury in acute TLS.

Moreover, precipitation of xanthine, less soluble in urine than uric acid, or other purine metabolites increased by use of allopurinol, may be rarely involved in the development of ARF (LaRosa et al 2007).

Oliguria due to acute uric acid nephropathy rapidly responds to hemodialysis (HD) often with a re-start of 
diuresis as the plasma uric acid level falls to $10 \mathrm{mg} / \mathrm{dL}$. Frequently the development of ARF in the cancer patient is enhanced by several factors that may induce a decrease in glomerular filtration, such as fluid depletion because of poor intake, fever and vomiting, and presence of renal dysfunctions due to tumor infiltration.

\section{Drugs for treatment of hyperuricemia in TLS}

\section{Allopurinol}

Allopurinol has been used in the US since 1964. Allopurinol is a structural isomer of hypoxanthine and acts to inhibit xanthine oxidase. This enzyme is responsible for the successive oxidation of hypoxanthine and xanthine resulting in the production of uric acid, the end-product of human purine metabolism. In addition to blocking uric acid production, inhibition of xanthine oxidase increases plasma concentrations of the uric acid precursors hypoxanthine and xanthine (Figure 1), which are converted to closely related purine ribotides adenosine and guanosine monophosphates. Increased levels of these ribotides causes feedback inhibition of amidophosphoribosyl transferase, the first and rate-limiting enzyme of purine biosynthesis (Pea 2005).

Allopurinol therefore decreases both uric acid formation and purine synthesis. Patients with or at high risk of tumor lysis still need to excrete the pre-existing uric acid that is not targeted by allopurinol.

Allopurinol is rapidly metabolized by its target, xanthine oxidase, to its active metabolite oxypurinol, which is also an inhibitor of xanthine oxidase. Allopurinol is almost completely metabolized to oxypurinol within 2 hours of oral administration, whereas oxypurinol is slowly excreted by the kidney over 18-30 hours (Holdsworth and Nguyen 2003).

Although allopurinol is effective in preventing further synthesis of uric acid, it has a limitation not being able to degrade the uric acid already present. Allopurinol is usually given together with alkaline hyperdiuresis to clear renal acid crystals, a process that takes up to 7-10 days; therefore its use during severe hyperuricemia may possibly imply a delay in starting chemotherapy. In fact chemotherapy should be instituted carefully in patients with hyperuricemia for at least 1-2 days after allopurinol therapy is initiated to allow uric acid to be excreted. Moreover, since xanthine and hypoxanthine are less soluble than uric acid, their accumulation may be toxic and may precipitate in a manner similar to that of uric acid, also causing renal failure In children with ALL receiving allopurinol, xanthine may predispose them to renal medullary sludging, which has been implicated as a possible mechanism for ARF (Andreoli et al 1986).
Side effects of allopurinol are rare, though significant when they occur; skin reactions are the most frequent adverse event reported, and antibiotics may cause an increase in these rashes. Serious skin reactions such as exfoliative rashes may occur as part of generalized hypersensitivity consisting of fever, skin rash, eosinophilia, hepatitis, worsening renal function and may result in toxic epidermal necrolysis or in Stevens-Johnson syndrome (Andreoli et al 1986). Hepatic complications may also occur in association with the hypersensitivity syndrome. The dose of allopurinol should be reduced in patients with impaired renal function.

Enhanced bone marrow suppression has been reported when allopurinol is used with cyclophosphamide and other cytotoxic agents (Cheson and Dutcher 2005). Allopurinol also inhibits the degradation of 6-mercaptopurine and azathioprine, thereby increasing their toxicity.

\section{Recombinant urate oxidase}

Urate oxidase is an enzyme involved in purine metabolism and is responsible for the oxidation of uric acid to allantoin, which has been shown to be 5-10 times more soluble in urine than uric acid. This enzyme is found in most mammals, but not in humans, because of a nonsense mutation in the coding region of the gene during hominoid evolution (Friedman et al 1985; Yeldandi et al 1991). Uric acid is the end product of purine metabolism in humans (Figure 2) (Cammalleri and Malaguarnera 2007).

The first molecule of this type, synthesized in 1968 and introduced in France and in Italy in 1975 and 1982, respectively, was a non-recombinant urate oxidase. It was a natural uricase, obtained from Aspergillus flavus cultures (Uricozyme ${ }^{\mathrm{TM}}$ ), indicated to prevent and treat hyperuricemia occurring during chemotherapy (Navolanic et al 2003) (Masera et al 1982).

The genetic absence of this molecule in humans and its proteic nature together with poor accuracy in purification and a slow production process confer a high immunogenicity to the compound, leading to elevated rate of hypersensivity reactions; $5 \%$ of patients developed reactions such as rashes, urticaria, angioedema, and bronchospasm within few minutes of infusion during the first administration (Pui et al 1997).

In 1996, using the recombinant DNA technique, another molecule was obtained from a genetically modified Saccharomyces cerevisiae strain that expresses urate oxidase cDNA, cloned from a strain of $A$. flavus (Leplatois et al 1992). This technology enabled production of a molecule of urate oxidase (rasburicase) characterized by higher purity and higher activity in compared with non-recombinant urate oxidase (Bayol et al 2002). 


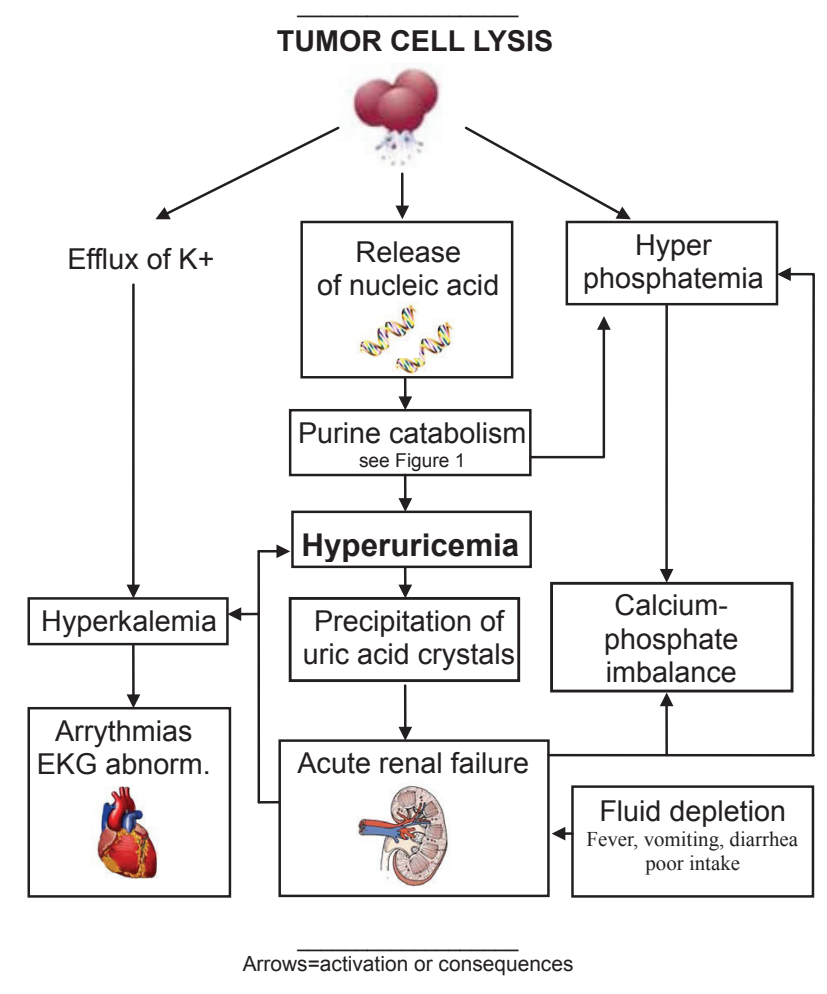

Figure 2 Pathogenesis of tumor lysis syndrome.

Generally, rasburicase maintains the same mechanism of action as the non-recombinant form of urate oxidase, but simply shows a significantly lower reaction rate. Even if allo-antibodies are documented in vivo in some reports, a lower immunogenicity of the compound is evident considering that other studies did not identify allo-antibodies even after several days of treatment (Goldman et al 2001). Pegylation tecnique has been proposed to prolong the halflife of rasburicase. Rasburicase use for treatment of other hyperuricemic conditions such as chronic gout is difficult, essentially because this drug is immunogenic and requires a daily administration.

\section{Pharmacokinetics of rasburicase}

Information about pharmacokinetics is derived from the use of rasburicase in children and young adults. Few data are available in adults and the elderly (Ueng 2005). The pharmacokinetics of rasburicase were studied in 40 patients with or at high risk of hyperuricemia enrolled in 2 clinical trials (Mahmoud et al 1998; Pui et al 2001a).

Rasburicase 0.15 or $0.2 \mathrm{mg} / \mathrm{kg}$ was administered intravenously over 30 minutes for 5-7 days. The mean $\mathrm{AUC}_{0-24}$ at $0.15 \mathrm{mg} / \mathrm{kg}$ was $32.9 \mathrm{mg} / \mathrm{L} / \mathrm{h}$ on day 1 and $34.4 \mathrm{mg} / \mathrm{L} / \mathrm{h}$ on day 5. The $\mathrm{AUC}_{0-24}$ was $45.2 \mathrm{mg} / \mathrm{L} / \mathrm{h}$ on day 1 and 47.3 $\mathrm{mg} / \mathrm{L} / \mathrm{h}$ on day 5 for the $0.2 \mathrm{mg} / \mathrm{kg}$ dose (Pui et al 2001a). At either dosage level, there was no significant difference between the mean $\mathrm{AUC}_{0-24}$ on days 1 and 5 indicating that rasburicase does not accumulate in the plasma. The mean peak concentrations on day 5 were 3.36 and $4.5 \mathrm{mg} / \mathrm{L}$ with 0.15 and $0.2 \mathrm{mg} / \mathrm{kg}$, respectively. At a dose of 0.15 and $0.2 \mathrm{mg} / \mathrm{kg}$, the mean half-life values were between 16-17.4 and 21.1 hours respectively For an half-life of 19 hours, rasburicase has to be administered once a day. Even after 5 days of treatment, a consistent accumulation has not been reported (Pui et al 2001a; Mahmoud et al 1998).

In vitro interaction studies show that rasburicase does not interact with allopurinol, cytarabine, methylprednisolone, methotrexate, mercaptopurine, thioguanine, etoposide, daunorubicin, cyclophosphamide, or vincristine (Pea 2005).

Association between rasburicase and allopurinol should be avoided, because the latter may reduce the effect of rasburicase owing to its inhibition of xanthine oxidase and consequent reduced uric acid concentration (Jeha and Pui 2005). As other protein rasburicase catabolism occurs by peptide hydrolysis without involvement of liver function, the cytochrome P450 is not induced or inhibited. For this reason coexistent hepatic pathology does not necessitate any adjustment of dosage (Navolanic et al 2003). Its clearance does not depend on renal function.

Because rasburicase causes enzymatic degradation of uric acid within blood/plasma/serum samples at room temperature, a falsely low plasma uric acid assay reading may be produced. Therefore, proper sample handling must be followed to avoid ex vivo uric acid degradation. Blood must be collected into pre-chilled tubes containing heparin and immediately immersed in an iced water bath. The plasma samples must be prepared by centrifugation in a pre-cooled centrifuge $\left(4^{\circ} \mathrm{C}\right)$ and maintained in an iced water bath and analyzed for uric acid within 4 hours of collection (Lim et al 2003).

\section{Safety profile}

Overall, rasburicase is well tolerated. The most common adverse event (AE) is skin rash, which was reported with an incidence of $2.5 \%$ in 196 patients evaluated in 3 trials. Skin rash was of grade I severity in 4 patients and was transient and localized in 1 patient. In another clinical trial evaluating 131 patients who were $<20$ years of age, $1(0.8 \%)$ severe $\mathrm{AE}$ (SAE) was registered: a 13-year-old girl with B-cell precursor ALL and pneumonia developed bronchospasm and hypoxemia 3 hours after the first dose of rasburicase and required treatment with oxygen and bronchodilator, and recovered 3 days later (Pui et al 2001a). Other AE included mild nausea 
and vomiting. Other adverse reactions are in order of decreasing incidence: fever $(6.8 \%)$, neutropenia with fever $(4 \%)$, respiratory distress $(3 \%)$, sepsis $(3 \%)$, neutropenia $(2 \%)$, mucositis $(2 \%)$, nausea $(1.7 \%)$, vomiting $(1.4 \%)$, headache $(0.9 \%)$, diarrhea $(0.9 \%)$, and abdominal pain (Cammalleri and Malaguarnera 2007).

Since patients with glucose-6-phosphate dehydrogenase (G6PD) deficiency are unable to break down hydrogen peroxide, a product of the urate oxidase reaction, urate oxidase is contraindicated in these patients for the risk of hemolysis (Browning and Kruse 2005).

However, hemolysis may occur in patients without G6PD deficiency. Rasburicase was discontinued because of hemolysis in an 11-year-old boy with pre-B precursor ALL and no evidence of G6PD deficiency (Goldman et al 2001).

The Food and Drug Administration has issued a Black Box Warning for rasburicase for hemolysis due to G6PD, hypersensitivity reactions including anaphylaxis, methemoglobinemia, and interference with uric acid measurements.

The formation of antibodies to rasburicase raises the concern that the administration of subsequent doses would increase the risk of hypersensitivity and decrease its efficacy. Antibodies against rasburicase or a part of it that is recognized by the immune system (epitopes) were detected in between $0 \%$ (Goldman et al 2001) and 14\% of patients (Pui et al 2001b).

Repeated use of rasburicase increases risk of hypersensitivity reactions: skin rashes (1.4\%), urticaria, bronchospasm $(<1 \%)$, dyspnea, hypoxemia, and anaphylactic shock $(<1 \%)$ (Jeha and Pui 2005). In these conditions, patients should be monitored during treatment and the drugs should be discontinued immediately, in association with an appropriate anti-allergic supportive therapy. Caution should be used in patients with a history of allergy. A re-treatment has the same efficacy but titers of antirasburicase antibodies (10\%-20\%) could increase, even though most of them are not neutralizing (Pui 2002).

Antibodies develop about 1-6 weeks after administration. The detection of antibodies is highly dependent on the sensitivity and specificity of the assay adopted for their determination and influenced by other factors such as serum sampling, timing and methodology, concomitant medications, and underlying disease. The presence of antibodies did not correlate with the clinical status of the patients or the occurrence of SAE/AE. The need for a re-treatment is rarer in neoplastic relapse, because this condition is more resistant to chemotherapy and so at lower risk of TLS development (Pui et al 2001b).

\section{Dosing and administration}

Rasburicase recommended dose is $0.20 \mathrm{mg} / \mathrm{kg} /$ day diluted in $50 \mathrm{~mL}$ of sodium chloride solution $(0.9 \%)$, administered intravenously for 30 minutes, daily for 3-5 days. Administration beyond 5 days is not recommended by the manufacturer, but a shorter period and/or lower dosage has been evaluated in a number of studies (Hummel et al 2005). A single dose of rasburicase, at low dosage, has shown a rapid reduction of hyperuricemia (Liu et al 2005; Hutcherson et al 2006; McDonnell et al 2006; Trifilio et al 2006). Contemporary use of alkalinization, hydration, and rasburicase at $0.10 \mathrm{mg} / \mathrm{kg}$ for 3-5 days maintains the same efficacy (Tarella et al 2005). In a recent review Cammalleri and Malaguarnera state that it may be more favorable to adjust the dose of rasburicase, according to clinical condition, type of malignancy, and chemotherapy adopted, in order to reduce the total cost of the patient's management and to reduce the risk of antibody development (Cammalleri and Malaguarnera 2007). Administration of more than one course of rasburicase is feasible, even if not recommended, because of the possible higher rate of reaction and/or antibodies mediated by drug inactivation.

Chemotherapy may begin 4-24 hours after completion of the first dose of rasburicase. Each vial of rasburicase must be reconstituted in the diluent provided by the producer, then diluted in $50 \mathrm{~mL}$ of preservative-free $\mathrm{NaCl} 0.9 \%$ and infused over 30 minutes within 24 hours of reconstitution.

\section{Monitoring}

Laboratory monitoring parameters should include serum uric acid, potassium, phosphate, calcium, and creatinine. Patients should be observed for allergic reactions. Symptoms may include urticaria, chest pain, and/or hypotension. Patients should also be monitored for any signs and symptoms of hemolysis or methemoglobinemia.

\section{Therapeutic issues}

Rasburicase was approved in the EU under the name Fasturtec $^{\circledR}$ in February 2001 and in the US under the name of Elitek ${ }^{\circledR}$ in July 2002 with slightly different indications: for the treatment and prevention of chemotherapy-induced hyperuricemia and ARF in patients with hematological malignancies at risk of TLS, and for the initial management of plasma uric acid levels in pediatric patients with leukemia, lymphoma, and solid tumor malignancies who are receiving anti-cancer therapy expected to result in tumor lysis and subsequent elevation of plasma uric acid, respectively. 


\section{Clinical studies on rasburicase}

The clinical development of rasburicase has been conducted primarily in Europe, the US, and Canada, and studies have accrued on more than 1200 subjects. Studies to date have included a phase I clinical trial, two phase II studies, a phase III comparative trial with allopurinol, and a safety study (Mahmoud et al 1998; Goldman et al 2001; Pui et al 2001a). In addition, compassionate-use trials in selected patients were conducted in Europe and North America prior to market availability (Pui et al 2001a; Patte et al 2002; Pession and Barbieri 2005).

\section{Phase I trials}

Rasburicase was first evaluated in single daily dose with dose escalation administered to 28 healthy male volunteers enrolled in a phase I, single-center, open-label trial (Mahmoud et al 1998). Four volunteers at each dosage level received either $0.05,0.1,0.15$, or $0.2 \mathrm{mg} / \mathrm{kg}$ intravenously in the first phase of the trial. A rapid dose-related decline in uric acid was observed: at 8 hours following a single IV infusion of rasburicase, uric acid levels decreased by $78.4 \%$, $84.3 \%, 91.9 \%$, and $96.0 \%$ with doses of $0.05,0.10,0.15$ $\mathrm{mg} / \mathrm{kg}$, and $0.20 \mathrm{mg} / \mathrm{kg}$, respectively. In the second phase, a daily dose of $0.1,0.15$, or $0.2 \mathrm{mg} / \mathrm{kg}$ was administered intravenously to 4 subjects at each dosage level for 5 consecutive days, confirming the rapid dose-related efficacy of the drug.

\section{Phase II trials}

In another study evaluating the efficacy and safety of rasburicase in 107 patients with NHL, ALL, or AML, rasburicase $0.15 \mathrm{mg} / \mathrm{kg} / \mathrm{d}$ was given for 5-7 days (Mahmoud et al 1998). Efficacy was determined by control of plasma uric acid concentration, with an upper limit of $6.5 \mathrm{mg} / \mathrm{dL}$ in patients $<13$ years of age or $7.5 \mathrm{mg} / \mathrm{dL}$ in patients $>13$ years old, and prevention of renal complications. Uric acid concentrations were reduced by at least $25 \% 4$ hours after the first dose in $94 \%$ and were below the upper limit $(6.5 \mathrm{mg} / \mathrm{dL}$ in patients $<13$ years of age or $7.5 \mathrm{mg} / \mathrm{dL}$ in patients $>13$ years old) by 48 hours in $99 \%$ of treated patients. Twenty-three patients experienced TLS, but none required dialysis. Approximately $7 \%$ of patients developed antibodies with inhibiting activity. Overall, rasburicase was well tolerated, with only 2 patients developing grade I rashes.

Pui et al conducted a pivotal multicenter, open-label, phase II clinical trial that enrolled 131 patients aged $<20$ years at high risk for or with hyperuricemia as a result of B mature or B-cell precursor or T-cell ALL, stage
III or IV Burkitt's lymphoma, or AML. All patients had either laboratory evidence of hyperuricemia (defined as a plasma uric acid concentration of $>8 \mathrm{mg} / \mathrm{dL}$ ) or were considered to be at high risk for hyperuricemia because of a large tumor burden. The study was divided into 2 phases: a dose-validation phase to select the optimally effective dose followed by an accrual phase, which verified the effectiveness of the dose chosen. The primary objectives of the study were to assess the efficacy, safety, and tolerability of rasburicase in controlling and normalizing malignancy-related or tumor lysis-related hyperuricemia. The secondary objective was to determine the pharmacokinetics of rasburicase in this population. The starting dose was $0.15 \mathrm{mg} / \mathrm{kg}(\mathrm{n}=12)$ and was increased by increments of $0.05 \mathrm{mg} / \mathrm{kg}$ up to $0.20 \mathrm{mg} / \mathrm{kg} / \mathrm{d}(\mathrm{n}=119)$, defined as the dose that corrected hyperuricemia (plasma uric acid $>6.5$ $\mathrm{mg} / \mathrm{dL}$ in patients $<13$ years old or $>7.5 \mathrm{mg} / \mathrm{dL}$ in older patients) within $48 \pm 2$ hours (mean \pm SD) after the start of treatment and prevented hyperuricemia for up to 24 hours after treatment in 14 consecutive patients. The dose was administered daily for 5-7 days, and the frequency could be increased to every 12 hours during the first 48 hours. The patient population was followed for up to 66 days. Around $50 \%$ of patients presented with hyperuricemia at onset. Overall, rasburicase produced a rapid and consistent decrease in plasma uric acid concentration in all patients treated with either dose. The median plasma uric acid concentration at diagnosis was $5.7 \mathrm{mg} / \mathrm{dL}$ (range 2.6-33.8) and declined to a median of $0.5 \mathrm{mg} / \mathrm{dL}$ (range $0.08-15.4$ ) 4 hours after the first rasburicase treatment $(\mathrm{p}<0.0001)$ both in 65 patients with hyperuricemia and in 66 patients without hyperuricemia at presentation. The median plasma uric acid remained at or near $0.5 \mathrm{mg} / \mathrm{dL}$ in both groups throughout the treatment course. Serum creatinine concentrations decreased significantly after 1 day of treatment in both groups and returned to normal range in all patients by day 6 . None of the patients required dialysis. Serum phosphorus concentrations, monitored daily, did not decrease until day 4 of treatment and returned to normal 48 hours after the end of treatment; meanwhile serum potassium concentrations remained stable throughout the treatment course. Rasburicase was well tolerated and only minimal toxicity was reported. The investigators suggested that rasburicase $0.2 \mathrm{mg} / \mathrm{kg}$ be used in future studies because 1 patient with stage III non-cleaved-cell NHL and an initial uric acid concentration of $21.1 \mathrm{mg} / \mathrm{dL}$ who received rasburicase $0.15 \mathrm{mg} / \mathrm{kg}$ had a transient increase in uric acid. In addition, rasburicase $0.2 \mathrm{mg} / \mathrm{kg}$ produced a higher rate 
of urinary excretion of allantoin; and no additional adverse effects were observed (Pui et al 2001a).

\section{Phase III trials}

Goldman et al reported an open-label, multicenter, randomized phase III trial conducted in the US between November 1996 and December 1997, comparing allopurinol with rasburicase for prophylaxis and treatment of hyperuricemia in children with leukemia or lymphoma at high risk for TLS. Patients were stratified by the presence or absence of hyperuricemia and by disease being eligible for the study if they had Murphy stage III or IV NHL, ALL with a peripheral white blood cell count $\geq 25 \times 10^{3} / \mathrm{mm}^{3}$ at presentation, or any childhood lymphoma or leukemia with a uric acid level $\geq 8 \mathrm{mg} / \mathrm{dL}$ at the time of study entry. Out of the 52 patients were enrolled: 20 patients with leukemia and 7 with lymphoma were randomized to receive for at least 5-7 days rasburicase $(0.2 \mathrm{mg} / \mathrm{kg}$ intravenously administered over 30 minutes daily); 19 patients with leukemia and 6 with lymphoma were randomized to receive oral allopurinol (100 $\mathrm{mg} / \mathrm{m}^{2}$ per dose or $3.3 \mathrm{mg} / \mathrm{kg}$ per dose administered every 8 hours). All patients received hyper-hydration of approximately $3 \mathrm{~L} / \mathrm{m}^{2}$ daily and intravenous sodium bicarbonate 20-40 mEq/L. Plasma uric acid was measured immediately before administration and at hours 4 and 12, then every 12 hours through 96 hours. The primary objective was to compare the decrease in mean plasma uric acid levels induced by the two drugs during the first 5 days of chemotherapy. The secondary objective was to compare the reduction of uric acid levels at 4 hours after administration of rasburicase prior to the delivery of chemotherapy. In the rasburicase group, 10 of the 27 patients were hyperuricemic prior to treatment; in the allopurinol group, 9 of the 25 patients were hyperuricemic at baseline. The two drugs were compared by measuring the mean uric acid AUC (area under the curve) from baseline to 96 hours $\mathrm{AUC}_{0-96}$. In the intent-to-treat analysis, the mean uric acid $\mathrm{AUC}_{0-96}$ was $128 \pm 70 \mathrm{mg} / \mathrm{dL} / \mathrm{h}$ for the rasburicase group and $329 \pm 129 \mathrm{mg} / \mathrm{dL} / \mathrm{h}$ for the allopurinol group ( $<<0.0001)$. Rasburicase-treated patients experienced a 2.6-fold lower exposure to plasma uric acid during the first 96 hours of therapy. Twenty-four hours after the first dose, patients randomized to receive rasburicase achieved a reduction in initial plasma uric acid levels of $86 \%$ compared with $12 \%$ for allopurinol ( $p<0.0001)$. Rasburicase therapy prevented further increases in serum creatinine levels, although the study size was too small to detect a significant difference in the incidence of ARF or in the number of patients requiring renal supportive measures; a single patient on the allopurinol arm, compared with none treated with rasburicase, required dialysis. No anaphylactic events occurred and no antibodies were detected at day 14 in the patients treated with rasburicase (Goldman et al 2001).

\section{Retrospectives and compassionate studies}

Patte et al conducted a retrospective review of 410 patients with advanced stage B-cell NHL and L3 ALL at the initiation of chemotherapy with the LMB 89 protocol, which has been followed in France since 1981. It consisted of 1 week of a cytoreductive low-dose regimen of chemotherapy concomitant with non-recombinant urate oxidase (Uricozyme, which is not widely available in the US) administered as an IV for 30 minutes for 5 days. Intensive chemotherapy was then initiated on day 8 . Only $5 \%$ of patients experienced severe metabolic problems after the initiation of therapy, and only $1.7 \%$ required dialysis as a result of TLS. The authors compared their data with published results from UKCCSG and POG protocols, which include essentially the same chemotherapy without the administration of urate oxidase. They noted that $16 \%$ of patients on the UKCCSG protocol and $23 \%$ of patients on the POG protocol required dialysis. This retrospective comparison suggested that treatment with urate oxidase may reduce metabolic and renal complications and the need for dialysis in these patients (Patte et al 2002).

In a large compassionate-use study, 245 patients (173 children, 72 adults) with cancer who had or were at high risk for hyperuricemia were enrolled (Pui et al 2001a). Rasburicase $0.2 \mathrm{mg} / \mathrm{kg}$ per dose was administered for 1-7 days and could be given every 12 hours for the first 72 hours in patients who were receiving chemotherapy and were at great risk of developing TLS. Hyperuricemia was defined as a uric acid concentration $>6.4,5.9,6.4,7.2$, or $6.4 \mathrm{mg} / \mathrm{dL}$ in patients aged $0-2,2-12,12-14,>14$ (males), or $>14$ (females) years, respectively. The most common diagnosis in the pediatric group was ALL (71\%). AML (33\%), NHL (26\%), and ALL $(18 \%)$ were the most frequent diagnoses in the adult group. Rasburicase was given to 117 pediatric and 49 adult patients as treatment for hyperuricemia; 56 pediatric and 23 adult patients received rasburicase as prophylaxis. There was a marked and constant decline in uric acid from a median of $9.7 \mathrm{mg} / \mathrm{dL}$ (range $6.2-33.5$ ) at diagnosis to a mean of 0.6 $\mathrm{mg} / \mathrm{dL}$ (range $0-8.1$ ) after treatment in the pediatric group $(\mathrm{p}<0.001)$. Rasburicase produced a similar result in the adult group with a marked decline from a median of $11.9 \mathrm{mg} / \mathrm{dL}$ (range 7-24.3) at diagnosis to $0.7 \mathrm{mg} / \mathrm{dL}$ (range 0-5) after treatment $(\mathrm{p}<0.001)$. In the prophylaxis group, 79 patients 
(56 children, 23 adults) had a significant decline in uric acid levels. However, 10 patients required dialysis because of hyperphosphatemia, azotemia, or both. Four children and 5 adults experienced mild adverse reactions that were drug related or of unknown etiology.

In a limited series of patients treated in 8 AIEOP (Associazione Italiana Ematologia Oncologia Pediatrica) centers from December 2002 to July 2003, we evaluated safety and efficacy of rasburicase in treatment and prophylaxis of hyperuricemia in children with malignancies at risk for developing TLS (Pession and Barbieri, 2005). We retrospectively reviewed 26 consecutive children: 12 patients $(46.2 \%), 8$ BALL, 3 solid tumor, 1 NHL, had hyperuricemia (group 1, treatment group), whereas 14 patients (54\%), 7 NHL, 4 AML, 3 B-ALL had normal or moderately increased uric acid level (UAL) (group 2, prophylaxis group). Normal values were defined according age $(<5 ; 5-10 ;>10$ years $)$ and gender at onset. At presentation the mean $\mathrm{UAL} \pm \mathrm{SD}$ was $13.7 \pm 3.4 \mathrm{mg} / \mathrm{dL}$ and $4.0 \pm$ $1.7 \mathrm{mg} / \mathrm{dL}$ for patients in group 1 and group 2, respectively. Other metabolic abnormalities present at baseline were: serum creatinine $>1.3 \mathrm{mg} / \mathrm{dL}$ in 5 patients $(19 \%)$ and serum phosphate $>7.0 \mathrm{mg} / \mathrm{dL}$ in $3(11 \%)$. Rasburicase was administered in 19 patients at the first induction chemotherapy 0-48 hours before the initiation of antiblastic infusion and in 7 during chemotherapy for relapse at $0.20 \mathrm{mg} / \mathrm{kg} \mathrm{IV}$ daily for median duration of 4 days (1-11). All patients showed a significant $(\mathrm{p}<0.001)$ reduction of UAL $48 \mathrm{~h}$ after treatment (group 1: $0.71 \pm 0.64 \mathrm{mg} / \mathrm{dL}$, group 2: 1.18 $\pm 1.14 \mathrm{mg} / \mathrm{dL}$ ). Rasburicase produced a highly significant $(\mathrm{p}<0.001)$ decrease in UAL within $24 \mathrm{hrs}$ after the first injection (group 1: $0.51 \pm 0.39 \mathrm{mg} / \mathrm{dL}$, group 2:1.83 \pm 1.07 $\mathrm{mg} / \mathrm{dL}$ ) with a response rate of $100 \%$ and $93 \%$ respectively. Normalization of creatinine and phosphorus levels was obtained after 5 and 4 days respectively the beginning of treatment. Only a child with Wilms' tumor developed acute renal failure without requiring dialysis. Our experience confirms that rasburicase is a safe and high effective drug, capable of inducing a rapid and marked decrease in UAL within 24 hours of the first injection. Rasburicase was well tolerated in all patients. This would suggest its use (at the dosage of $0.20 \mathrm{mg} / \mathrm{kg}$ for at least 5 days) in children defined at high risk of TLS by clinical (ALL with leukemia/lymphoma syndrome, B-ALL FAB L3, stage III/IV NHL, bulky disease, renal impairment) or laboratory criteria (WBC $>$ $50 \times 109 / \mathrm{L}, \mathrm{LDH}>2$ times over normal range, $\mathrm{UAL}>7$ $\mathrm{mg} / \mathrm{dL}$, creatininemia or $\mathrm{Ccr}, \mathrm{cm} \times 0.55 /$ creatinemia, over normal range for age). Patients at low risk would be treated with standard therapy (allopurinol, hyper-hydration, and urine alkalinization). The steady improvement of renal function during treatment with rasburicase in patients having impaired renal function at presentation, none of whom required dialysis, is remarkable. The low incidence of ARF associated with the absence of toxicity suggests that this drug is cost-effective (Oldfield and Perry 2006).

\section{Prevention and therapy of TLS}

All adult and pediatric patients with hematological malignancy or solid tumors at risk of TLS deserve an adequate prophylaxis treatment; close monitoring of risk factors for a prompt identification of signs and symptoms are important factors in preventing TLS-related life-threatening events. In this section we propose a possible therapeutic schema in adults and pediatric patient for prevention of TLS, according to risk group (Table 3 ) and for treatment of both for laboratory and clinical TLS (Table 4).

Regrettably, this approach may be unsuccessful in controlling severe hyperuricemia, and death as a result of TLS-related complications occurred in $1 \%-2 \%$ of the overall population.

\section{Conclusions}

TLS is a potential complication in any patient with malignancies. It has been identified in a population of patients at higher risk of TLS which includes subjects affected by bulky or highly chemotherapy-sensitive tumors such as hematological malignancies, whereas it is more rarely observed spontaneously or as a consequence of treatment in adult and pediatric solid tumors.

Hallmarks of TLS are hyperuricemia, hyperkalemia, hyperphosphatemia, and hypocalcemia which may follow the massive lysis of tumor cells. Major complications of TLS are metabolic acidosis and acute renal failure. In view of its complex pathophysiology and clinical presentation, the myriad of metabolic disorders must be assessed and treated rapidly. Proper fluid management, careful alkalinization of the urine, correction of acidosis, inhibition of the formation of uric acid, or destruction of the already circulating uric acid molecules, as well as attention to the opportunity for starting antineoplastic treatments, are mainstays of therapy. Currently, allopurinol and rasburicase are the two drugs available for lowering serum uric acid. The advantages of rasburicase are its rapid onset thanks to its unique mechanism of action. It lowers pre-existing uric acid and prevents the further accumulation of uric acid and xantyne, therefore minimizing the risk of tumor lysis syndrome and ARF, while allopurinol does 
Table 3 Prevention of tumor lysis syndrome

\section{Pediatric patients}

Low risk - Allopurinol orally $300 \mathrm{mg} / \mathrm{m}^{2} /$ day or $10 \mathrm{mg} / \mathrm{kg} /$ day 3 times. - However a single dose of rasburicase will be take into account for also low-risk patients

- Hyperhydratation with glucosaline or normal saline solution $2.5-3 \mathrm{~L} / \mathrm{m}^{2} /$ day or $200 \mathrm{~mL} / \mathrm{kg} /$ day if children $<10 \mathrm{~kg}$ - Alkalinization $\mathrm{NaHCO}_{3} 50-70 \mathrm{mEq} / \mathrm{L}$ to maintain urinary $\mathrm{pH}>7$

- Furosemide if urine volume is $<100 \mathrm{~mL} / \mathrm{m}^{2} / \mathrm{h}$ or $3 \mathrm{~mL} / \mathrm{kg} / \mathrm{h}$ for children $<10 \mathrm{~kg}$

High risk - Rasburicase $0.2 \mathrm{mg} / \mathrm{kg} /$ day at least 4 hours before beginning chemotherapy for $3-5$ days

- Hyperhydratation with glucosaline or normal saline solution $2.5-3 \mathrm{~L} / \mathrm{m}^{2} /$ day or $200 \mathrm{~mL} / \mathrm{kg} /$ day if children $<10 \mathrm{~kg}$ - Furosemide if urine volume is $<100 \mathrm{~mL} / \mathrm{m}^{2} / \mathrm{h}$ or $3 \mathrm{~mL} / \mathrm{kg} / \mathrm{h}$ for children $<10 \mathrm{~kg}$

\section{Adult patients}

Low risk - Allopurinol orally $300 \mathrm{mg} / \mathrm{m}^{2} /$ day 3 times - Hyperhydratation $2.5-3 \mathrm{~L} / \mathrm{m}^{2} /$ day with glucosaline or normal saline solution. Hydratation should always be performed, except in patients at risk of rapid volume overload

- Alkalinization to maintain urine $\mathrm{pH}>7$

- Furosemide or mannitol if urine volume is $<100 \mathrm{~mL} / \mathrm{m}^{2} / \mathrm{h}$. Diuretics should be administered unless hypovolemia or obstructive uropathy are diagnosed to maintain urine outputof at least $100 \mathrm{~mL} / \mathrm{m}^{2} / \mathrm{h}$ with urinary specific gravity $\leq 1010$

High risk - Rasburicase $0.05-0.2 \mathrm{mg} / \mathrm{kg} /$ day for $3-5$ days

- Hyperhydratation 2.5-3 L/m²/day with glucosaline or normal saline solution. Hydratation should always be performed, except in patients at risk of rapid volume overload

- Furosemide or mannitol if urine volume is $<100 \mathrm{~mL} / \mathrm{m}^{2} / \mathrm{h}$. Diuretics should be administered unless hypovolemia or obstructive uropathy are diagnosed to maintain urine output of at least $100 \mathrm{~mL} / \mathrm{m}^{2} / \mathrm{h}$ with urinary specific gravity $\leq 1010$

- For solid tumors reduced chemotherapy schedule may be evaluated to reduce tumor burden and related TLS risk

Rasburicase is contraindicated in patients with metahemoglobinemia, G6PD deficiency or other metabolic disorders that may cause hemolitic anemia.

not lower pre-existing uric acid and has a delayed onset of action. On the other hand, rasburicase has no effect on the profound acid-base and electrolyte disturbances that can occur during TLS.

At the present time, rasburicase provides an alternative for the prevention of TLS in patients at high risk and it is strongly suggested for the treatment of hyperuricemia and TLS. This molecule should therefore be added to the formulary of hospital hematology and oncology clinics. However, there should be some restrictions for its use according to recommendations and guidelines to define subgroups of
Table 4 Treatment of laboratory tumor lysis syndrome (LTLS and clinical tumor lysis syndrome (CTLS)

\section{Laboratory TLS pediatric patients}

Low risk - Allopurinol orally $300 \mathrm{mg} / \mathrm{m}^{2} /$ day or $10 \mathrm{mg} / \mathrm{kg} /$ day 3 times.

- However a single dose of rasburicase will be take into account for also low-risk patients

- Hyperhydratation with glucosaline or normal saline solution $2.5-3 \mathrm{~L} / \mathrm{m}^{2} /$ day or $200 \mathrm{~mL} / \mathrm{kg} /$ day if children $<10 \mathrm{~kg}$

- Urine alkalinization $\mathrm{NaHCO}_{3} 50-70 \mathrm{mEq} / \mathrm{L}$ to maintain urinary $\mathrm{pH}>7$

- Furosemide if urine volume is $<100 \mathrm{~mL} / \mathrm{m}^{2} / \mathrm{h}$ or $3 \mathrm{~mL} / \mathrm{kg} / \mathrm{h}$ for children $<10 \mathrm{~kg}$

High risk - Rasburicase $0.2 \mathrm{mg} / \mathrm{kg} /$ day. at least 4 hours before beginning chemotherapy for $3-5$ days

- Hyperhydratation with glucosaline or normal saline solution $2.5-3 \mathrm{~L} / \mathrm{m}^{2} /$ day or $200 \mathrm{~mL} / \mathrm{kg} /$ day if children $<10 \mathrm{~kg}$

- Furosemide if urine volume is $<100 \mathrm{~mL} / \mathrm{m}^{2} / \mathrm{h}$ or $3 \mathrm{~mL} / \mathrm{kg} / \mathrm{h}$ for children $<10 \mathrm{~kg}$

\section{Clinical TLS pediatric patients}

- Rasburicase $0.2 \mathrm{mg} / \mathrm{kg} /$ day at least 4 hours before beginning chemotherapy for 3-5 days.

- Hyperhydratation with glucosaline or normal saline solution $2.5-3 \mathrm{~L} / \mathrm{m}^{2} /$ day or $200 \mathrm{~mL} / \mathrm{kg} /$ day if children $<10 \mathrm{~kg}$.

- Furosemide if urine volume is $<100 \mathrm{~mL} / \mathrm{m}^{2} / \mathrm{h}$ or $3 \mathrm{~mL} / \mathrm{kg} / \mathrm{h}$ for children $<10 \mathrm{~kg}$.

- Consider hemodialysis.

\section{Laboratory and clinical TLS adult patients}

There is no major differences in treatment of laboratory and clinical TLS in adult patients

- Rasburicase $0.2 \mathrm{mg} / \mathrm{kg} /$ day for 3-5 days.

- Hyperhydratation 2.5-3 L/m²/day with glucosaline or normal saline solution. Hydratation should always be performed, except in patients at risk of rapid volume overload.

- Furosemide or mannitol if urine volume is $<100 \mathrm{~mL} / \mathrm{m}^{2} / \mathrm{h}$. Diuretics should be administered unless hypovolemia or obstructive uropathy are diagnosed to maintain urine output of at least $100 \mathrm{~mL} / \mathrm{m}^{2} / \mathrm{h}$ with urinary specific gravity $\leq 1010$.

- Low dose dopamine can be used to improve renal perfusion.

- Consider hemodialysis.

Rasburicase is contraindicated in patients with metahemoglobinemia, G6PD deficiency or other metabolic disorders that may cause hemolitic anemia.

patients that would benefit from its use. Presently, there is a lack of pharmacoeconomic data comparing rasburicase and oral and/or intravenous allopurinol in the areas of delays in chemotherapy treatment and effects on renal function. Additionally, clinical data do not yet provide enough evidence to support replacing allopurinol with rasburicase as the standard of care in patients not at high risk of TLS or with laboratory TLS.

Moving towards more extensive and intensive antineoplastic strategies, both for children and elderly, the availability of a rapid and strongly effective drug such as 
rasburicase, to control and manage hyperuricemia and TLS, appears a valuable and safe tool that should be well known and always considered at onset for any cancer patient.

\section{References}

Alkhuja S, Ulrich H. 2002. Acute renal failure from spontaneous acute tumor lysis syndrome: a case report and review. Ren Fail, 24:227-32.

Andreoli SP, Clark JH, McGuire WA, et al. 1986. Purine excretion during tumor lysis in children with acute lymphocytic leukemia receiving allopurinol: relationship to acute renal failure. $J$ Pediatr, 109292-8.

Annemans L, Moeremans K, Lamotte M, et al. 2003. Incidence, medical resource utilisation and costs of hyperuricemia and tumour lysis syndrome in patients with acute leukaemia and non-Hodgkin's lymphoma in four European countries. Leuk Lymphoma, 44:77-83.

Barton JC. 1989. Tumor lysis syndrome in nonhematopoietic neoplasms. Cancer, 64:738-40.

Bayol A, Capdevielle J, Malazzi P, et al. 2002. Modification of a reactive cysteine explains differences between rasburicase and Uricozyme, a natural Aspergillus flavus uricase. Biotechnol Appl Biochem, 36:21-31.

Bedrna JPJ. 1929. Akuter harnleiterverschluss nach bestrahlung chronischer leukamien mit rontgenstrahlen. Med Klin, 25:1700-1.

Browning LA, Kruse JA. 2005. Hemolysis and methemoglobinemia secondary to rasburicase administration. Ann Pharmacother, 39:1932-5.

Cairo MS, Bishop M. 2004. Tumour lysis syndrome: new therapeutic strategies and classification. Br J Haematol, 127:3-11.

Cammalleri L, Malaguarnera M. 2007. Rasburicase represents a new tool for hyperuricemia in tumor lysis syndrome and in gout. Int J Med Sci, 4:83-93.

Cheson BD, Dutcher BS. 2005. Managing malignancy-associated hyperuricemia with rasburicase. J Support Oncol, 3:117-24.

Coutinho AK, De OSM, Pinczowski H, et al. 1997. Tumor lysis syndrome in a case of chronic lymphocytic leukemia induced by high-dose corticosteroids. Am J Hematol, 54:85-6.

Feld, J., Mehta, H, Burkes, RL. 2000. Acute spontaneous tumor lysis syndrome in adenocarcinoma of the lung: a case report. Am J Clin Oncol, 23, 491-3.

Filler G. 2001. Acute renal failure in children: aetiology and management. Paediatr Drugs, 3:783-92.

Flombaum CD. 2000. Metabolic emergencies in the cancer patient. Semin Oncol, 27:322-34.

Friedman TB, Polanco GE, Appold JC, et al. 1985. On the loss of uricolytic activity during primate evolution - I. Silencing of urate oxidase in a hominoid ancestor. Comp Biochem Physiol B, 81:653-9.

Goldman SC, Holcenberg JS, Finklestein JZ, et al. 2001. A randomized comparison between rasburicase and allopurinol in children with lymphoma or leukemia at high risk for tumor lysis. Blood, 97:2998-3003.

Habib GS, Saliba WR. 2002. Tumor lysis syndrome after hydrocortisone treatment in metastatic melanoma: a case report and review of the literature. Am J Med Sci, 323:155-7.

Holdsworth MT, Nguyen P. 2003. Role of i.v. allopurinol and rasburicase in tumor lysis syndrome. Am J Health Syst Pharm, 60:2213-22; quiz 2223-4.

Hsu HH, Chan YL, Huang CC. 2004. Acute spontaneous tumor lysis presenting with hyperuricemic acute renal failure: clinical features and therapeutic approach. J Nephrol, 17:50-6.

Hsu HH, Huang CC. 2004. Acute spontaneous tumor lysis in anaplastic large T-cell lymphoma presenting with hyperuricemic acute renal failure. Int J Hematol, 79:48-51.

Hummel M, Buchheidt D, Reiter S, et al. 2005. Recurrent chemotherapyinduced tumor lysis syndrome TLS. with renal failure in a patient with chronic lymphocytic leukemia - successful treatment and prevention of TLS with low-dose rasburicase. Eur J Haematol, 75:518-21.
Hutcherson DA, Gammon DC, Bhatt MS, et al. 2006. Reduced-dose rasburicase in the treatment of adults with hyperuricemia associated with malignancy. Pharmacotherapy, 26:242-7.

Jasek AM, Day HJ. 1994. Acute spontaneous tumor lysis syndrome. Am J Hematol, 47:29-31.

Jeha S, Pui CH. 2005. Recombinant urate oxidase rasburicase. in the prophylaxis and treatment of tumor lysis syndrome. Contrib Nephrol, 147:9-79.

Larosa C, McMullen L, Bakdash S, et al. 2007. Acute renal failure from xanthine nephropathy during management of acute leukemia. Pediatr Nephrol, 22:32-5.

Leplatois P, Le Douarin B, Loison G. 1992. High-level production of a peroxisomal enzyme: Aspergillus flavus uricase accumulates intracellularly and is active in Saccharomyces cerevisiae. Gene, 122:39-45.

Lim E, Bennett P, Beilby J. 2003. Sample preparation in patients receiving uric acid oxidase rasburicase therapy. Clin Chem, 49:417-9.

Liu CY, Sims-McCallum RP, Schiffer CA. 2005. A single dose of rasburicase is sufficient for the treatment of hyperuricemia in patients receiving chemotherapy. Leuk Res, 29:63-5.

Locatelli F, Rossi F. 2005. Incidence and pathogenesis of tumor lysis syndrome. Contrib Nephrol, 147:1-8.

Mahmoud HH, Leverger G, Patte C, et al. 1998. Advances in the management of malignancy-associated hyperuricaemia. Br J Cancer, 77(Suppl 4):8-20.

Masera G, Jankovic M, Zurlo MG, et al. 1982. Urate-oxidase prophylaxis of uric acid-induced renal damage in childhood leukemia. J Pediatr, 100:52-5.

McDonnell AM, Lenz KL, Frei-Lahr DA, et al. 2006. Single-dose rasburicase $6 \mathrm{mg}$ in the management of tumor lysis syndrome in adults. Pharmacotherapy, 26:806-12.

Navolanic PM, Pui CH, Larson RA, et al. 2003. Elitek-rasburicase: an effective means to prevent and treat hyperuricemia associated with tumor lysis syndrome, a Meeting Report, Dallas, Texas, January 2002. Leukemia, 17:499-514.

Oldfield V, Perry CM. 2006. Spotlight on rasburicase in anticancer therapyinduced hyperuricemia. BioDrugs, 20:197-9.

Parham WA, Mehdirad AA, Biermann KM, et al. 2006. Hyperkalemia revisited. Tex Heart Inst J, 33:40-7.

Patte C, Sakiroglu C, Ansoborlo S, et al. 2002. Urate-oxidase in the prevention and treatment of metabolic complications in patients with B-cell lymphoma and leukemia, treated in the Societe Francaise d'Oncologie Pediatrique LMB89 protocol. Ann Oncol, 13:789-95.

Pea F. 2005. Pharmacology of drugs for hyperuricemia. Mechanisms, kinetics and interactions. Contrib Nephrol, 147:35-46.

Pession A, Barbieri E. 2005. Treatment and prevention of tumor lysis syndrome in children. Experience of Associazione Italiana Ematologia Oncologia Pediatrica. Contrib Nephrol, 147:80-92.

Pui, CH. 2002. Rasburicase: a potent uricolytic agent. Expert Opin Pharmacother, 3:433-42.

Pui CH, Jeha S, Irwin D, et al. 2001a. Recombinant urate oxidase rasburicase. in the prevention and treatment of malignancy-associated hyperuricemia in pediatric and adult patients: results of a compassionate-use trial. Leukemia, 15:1505-9.

Pui CH, Mahmoud HH, Wiley JM, et al. 2001b. Recombinant urate oxidase for the prophylaxis or treatment of hyperuricemia in patients with leukemia or lymphoma. J Clin Oncol, 19:697-704.

Pui CH, Relling MV, Lascombes F, et al. 1997. Urate oxidase in prevention and treatment of hyperuricemia associated with lymphoid malignancies. Leukemia, 11:1813-6.

Ribeiro RC, Pui CH. 2003. Recombinant urate oxidase for prevention of hyperuricemia and tumor lysis syndrome in lymphoid malignancies. Clin Lymphoma, 3:225-32.

Riccio B, Mato A, Olson EM, et al. 2006. Spontaneous tumor lysis syndrome in acute myeloid leukemia: two cases and a review of the literature. Cancer Biol Ther, 5:1614-7.

Sparano J, Ramirez M, Wiernik PH. 1990. Increasing recognition of corticosteroid-induced tumor lysis syndrome in non-Hodgkin's lymphoma. Cancer, 65:1072-3. 
Stark ME, Dyer MC, Coonley CJ. 1987. Fatal acute tumor lysis syndrome with metastatic breast carcinoma. Cancer, 60:762-4.

Tarella C, Bono D, Zanni M, et al. 2005. Intensive chemotherapy in patients with lymphoma. Management of the risk of hyperuricemia. Contrib Nephrol, 147:93-104.

Tomlinson GC, Solberg LA Jr. 1984. Acute tumor lysis syndrome with metastatic medulloblastoma. A case report. Cancer, 53:1783-5.

Trifilio S, Gordon L, Singhal S, et al. 2006. Reduced-dose rasburicase recombinant xanthine oxidase. in adult cancer patients with hyperuricemia. Bone Marrow Transplant, 37:997-1001.

Turtureanu-Hanganu E. 2002. Emergency care: the tumor lysis syndrome. Rev Med Chir Soc Med Nat Iasi, 106:705-11.
Ueng S. 2005. Rasburicase Elitek.: a novel agent for tumor lysis syndrome. Proc Bayl Univ Med Cent, 18:275-9.

Wossmann W, Schrappe M, Meyer U, et al. 2003. Incidence of tumor lysis syndrome in children with advanced stage Burkitt's lymphoma/leukemia before and after introduction of prophylactic use of urate oxidase. Ann Hematol, 82:160-5.

Yeldandi AV, Yeldandi V, Kumar S, et al. 1991. Molecular evolution of the urate oxidase-encoding gene in hominoid primates: nonsense mutations. Gene, 109:281-4. 
